\title{
The Role of Emotion in Decision Making
}

\section{A Cognitive Neuroscience Perspective}

\author{
Nasir Naqvi, ${ }^{1}$ Baba Shiv, ${ }^{2}$ and Antoine Bechara ${ }^{3}$ \\ ${ }^{1}$ Division of Cognitive Neuroscience, Department of Neurology, University of Iowa College of Medicine ${ }^{2}$ Graduate School of \\ Business, Stanford University; and ${ }^{3}$ Brain and Creativity Institute, and Department of Psychology, University of Southern \\ California
}

\begin{abstract}
Decision making often occurs in the face of uncertainty about whether one's choices will lead to benefit or harm. The somatic-marker hypothesis is a neurobiological theory of how decisions are made in the face of uncertain outcome. This theory holds that such decisions are aided by emotions, in the form of bodily states, that are elicited during the deliberation of future consequences and that mark different options for behavior as being advantageous or disadvantageous. This process involves an interplay between neural systems that elicit emotional/ bodily states and neural systems that map these emotional/ bodily states.
\end{abstract}

KEYWORDS—decision making; frontal lobes; neuropsychology; neuroeconomics; emotion

Decision making precedes many of life's most important events: choosing whom to marry, which house to buy, which stock to invest in, whether to have just one more drink before hitting the road, whether to have surgery, and whether to quit smoking, to name a few examples. Properly executed decision making gives rise to some of the most elevated human abilities, such as ethics, politics, and financial reasoning. Derangements of decision making underlie some of the more tragic consequences of psychiatric illnesses such as drug addiction, eating disorders, obsessivecompulsive disorder, schizophrenia, mania, and personality disorders (Rahman, Sahakian, Cardinal, Rogers, \& Robbins, 2001).

The field of economics, which is concerned with formalizing the rules that govern human decision making, has begun to focus increasingly on forms of decision making that go beyond simple cost-benefit analysis. Traditional economic theory assumed that

Address correspondence to Antoine Bechara, Hedco Neuroscience Building, University of Southern California, Los Angeles, CA 900892520; e-mail: bechara@usc.edu. most decision making involves rational Bayesian maximization of expected utility, as if humans were equipped with unlimited knowledge, time, and information-processing power. The prevalent assumption of this view was that a direct link exists between knowledge and the implementation of behavioral decisions - that is, that one does what one actually knows. In the 1970s and 1980s, decision-making researchers identified phenomena that systematically violated such normative principles of economic behavior (see Kahneman \& Tversky, 1979). In the 1990 s, they began to show that many forms of decision making, especially those that involve a high level of risk and uncertainty, involve biases and emotions that act at an implicit level (see Hastie \& Dawes, 2001).

In recent years, decision making has become a subject of neuroscience research. Neuroscientists applying diverse methods, including the lesion method (using brain damage that occurs as a result of stroke, etc., to examine how different brain areas contribute to various mental functions), functional imaging, and other physiological techniques, have begun to elucidate the neural process underlying the execution of successful and unsuccessful decisions. This effort has converged with the field of behavioral economics in showing that decision making involves not only the cold-hearted calculation of expected utility based upon explicit knowledge of outcomes but also more subtle and sometimes covert processes that depend critically upon emotion. Here, we focus on a particular neurobiological theory of decision making, termed the somatic-marker hypothesis, in which emotions, in the form of bodily states, bias decision making toward choices that maximize reward and minimize punishment.

\section{INSIGHTS FROM PATIENTS WITH FOCAL BRAIN DAMAGE}

The modern era of the neuroscience of decision making began with the observation by Antonio Damasio that patients with 
damage in the ventromedial prefrontal cortex (vmPFC), an area of the brain located above the eye sockets, often engaged in behaviors that were detrimental to their well-being. The actions that these patients elected to pursue led to diverse kinds of losses including financial losses, losses in social standing, and losses of family and friends. These patients seemed unable to learn from previous mistakes, as reflected by repeated engagement in decisions that led to negative consequences. In striking contrast to this real-life decision-making impairment, these patients' intellect and problem-solving abilities were largely normal; their decision-making deficits could not be explained by impairments in the retrieval of semantic knowledge pertinent to the situation, language comprehension or expression, attention, working memory, or long-term memory (Damasio, 1994).

An important insight into the nature of the impairments resulting from vmPFC damage came from the observation that, in addition to their inability to make advantageous decisions in real life, patients with damage to the vmPFC evinced a generally flat affect, and their ability to react to emotional situations was somewhat impaired. This led Damasio to hypothesize that the primary dysfunction of patients with vmPFC damage was an inability to use emotions to aid in decision making, particularly decision making in the personal, financial, and moral realms. This was the fundamental tenet of the somatic-marker hypothesis: that emotions play a role in guiding decisions, especially in situations in which the outcome of one's choices, in terms of reward and punishment, are uncertain.

Testing the somatic-marker hypothesis required first devising a task that simulated the demands of real-life decision making by factoring in uncertain reward and punishment. This led one of us (Antoine Bechara) to develop what is now known as the Iowa Gambling Task (the details of this task and the results from lesion studies using it are reviewed in Bechara \& Damasio, 2005). In this task, subjects choose from four decks of cards that provide varying levels of reward and punishment (winning and losing play money). Two of the decks provide low reward, but also a low level of punishment. Choosing consistently from these decks eventually leads to a net gain of money; they are designated as "advantageous" decks. The other two decks provide a high reward, but also a high punishment. Choosing consistently from these decks eventually leads to a net loss of money; they are designated as "disadvantageous" decks.

In the Iowa Gambling Task, normal individuals initially sampled the advantageous and disadvantageous decks equally, but, after experiencing the high punishments from the disadvantageous decks, they shifted their choices to the advantageous decks. In contrast, subjects with vmPFC damage tended to continue choosing from the disadvantageous decks, seemingly insensitive to the negative consequences of this choice. This strategy mimicked the real-life impairments of these subjects.

The next step in testing the somatic-marker hypothesis was to address the role of emotions in decision making. According to the theory, emotions are constituted by changes in the body. These bodily states are elicited during the decision-making process and function to "mark" certain options as advantageous and other options as disadvantageous. To test this hypothesis, Bechara and colleagues coupled their gambling task with the measurement of skin-conductance response (SCR), an autonomic index of emotional arousal. In a series of experiments, it was shown that normal subjects elicited SCRs that were larger before choosing from the disadvantageous decks than before choosing from the advantageous decks. Furthermore, it was found that this anticipatory emotional response preceded explicit knowledge of the correct strategy. Patients with vmPFC damage, in contrast, did not show such anticipatory emotional responses. Importantly, vmPFCdamaged subjects had intact SCRs to receiving rewards and punishments, suggesting that the vmPFC is not necessary for registering the emotional impact of rewards and punishments after they are delivered. Rather, this region is necessary for anticipating the emotional impact of future rewards and punishment.

Further experiments showed that subjects with lesions in the amygdala, a medial-temporal-lobe region that is also known to be involved in emotion, also had impaired performance on the gambling task. Like patients with vmPFC damage, patients with amygdala damage also tended to choose more often from the disadvantageous decks. Also like patients with vmPFC damage, those with amygdala damage did not have anticipatory SCRs before choosing from the disadvantageous decks. However, unlike vmPFC-damaged subjects, these subjects also had impaired SCRs to receiving rewards and punishments. This suggested that subjects with amygdala damage had an impairment in registering the emotional impact of rewards and punishments caused by specific behaviors, a function necessary for being able to anticipate the rewarding and punishing consequences of these behaviors in the future.

This set of results gave rise to a model of decision making in which the amygdala and vmPFC play distinct but related roles (Fig. 1). The amygdala triggers emotional/bodily states in response to receiving rewards and punishments that are caused by specific behaviors. Through a learning process, these emotional/ bodily states become linked to mental representations of the specific behaviors that brought them about. During decision making, the subject deliberates these behaviors as options for the future. As each option is brought to mind, the somatic state that was triggered by that behavior in the past is reenacted by the vmPFC. After the emotional/bodily states are elicited in the body during decision making, they are represented in the brain through a sensory process. This can occur in two ways. The mapping of bodily/emotional states at the cortical level, such as within the insular cortex, gives rise to conscious "gut feelings" of desire or aversion that are attributed to specific behavioral options. The mapping of bodily states at the subcortical level, such as within the mesolimbic dopamine system, occurs in a nonconscious fashion, such that subjects choose the advantageous option without feeling specific feelings of desire for that option or aversion to the disadvantageous option. 


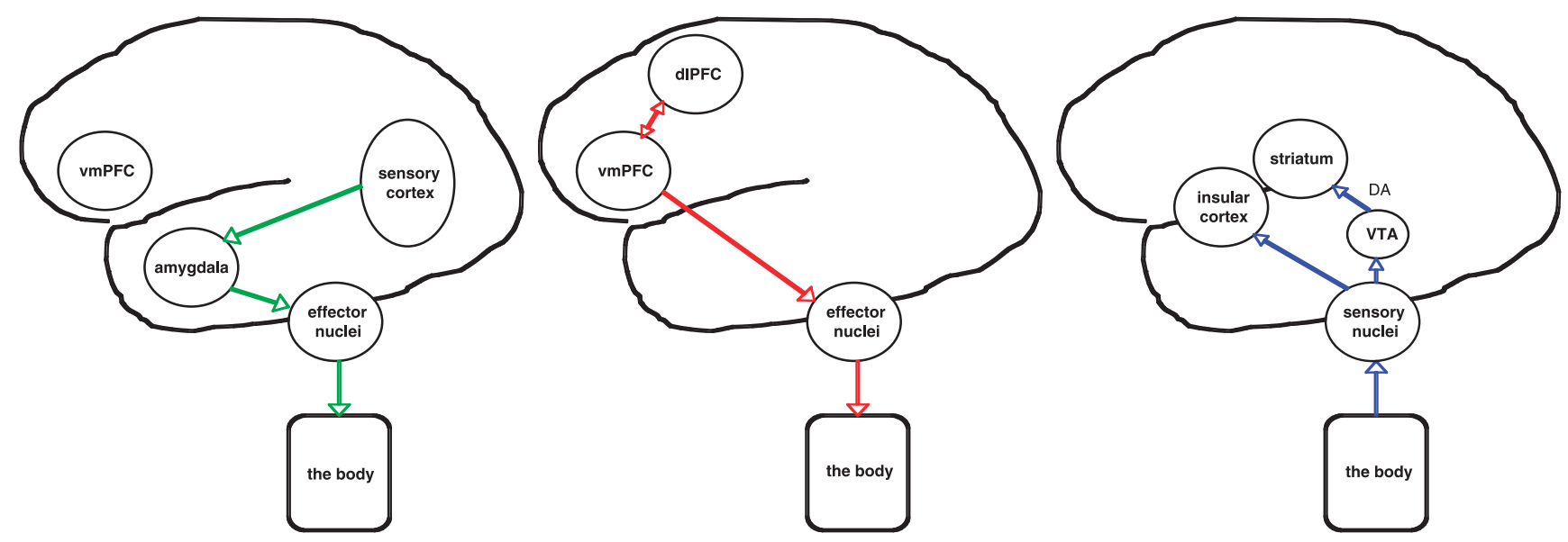

Fig. 1. Schematic model of somatic-state activation during reward-related decision making. First, sensory properties of rewards and punishments drive the amygdala to elicit emotional/bodily responses. This occurs through connections from higher-order cortices that represent the sensory properties of rewards (sensory cortex) to the amygdala to effector nuclei in the brain stem, which control bodily processes such as autonomic responses (left diagram). These responses become linked to internal representations in the ventromedial prefrontal cortex (vmPFC) of the specific behavioral choices that brought them about. During decision making, the vmPFC re-enacts these emotional/bodily states as the same behavioral choices are contemplated as options for the future. This occurs through connections between the dorsolateral prefrontal cortex (dIPFC), which is involved in holding mental representations of specific behaviors in mind, and the vmPFC (middle diagram). Emotional/bodily states elicited during decision making are then mapped within sensory systems (right diagram). The mapping of body states within the insular cortex gives rise to conscious "gut feelings" of desire or aversion that are attributed to specific behavioral options. Emotional/bodily states can also be mapped within the mesolimbic dopamine system, which includes the dopamine (DA) neurons within the ventral tegmental area (VTA) and their targets within the striatum. This latter process can bias decision making towards the advantageous choice in a nonconscious fashion.

\section{EVIDENCE FROM PHYSIOLOGICAL STUDIES}

At around the same time that Damasio and his colleagues were using lesion studies to address the role of the vmPFC in decision making, Edmund Rolls and his colleagues were exploring the reward-related functions of the vmPFC by recording the electrical activity of single neurons within this region (this work is reviewed in Rolls, 2004). They found that vmPFC neurons respond to the receipt of various primary reinforcers, such as palatable foods. Furthermore, they found that responses to specific primary reinforcers were reduced by manipulations that diminished their value, such as feeding someone a palatable food to satiety. In addition, they found that vmPFC neurons respond to conditioned stimuli that predict the delivery of primary reinforcers.

Functional imaging studies have extended these findings to humans. A functional magnetic resonance imagery (fMRI) study by Gottfried, O'Doherty, \& Dolan (2003) found that the responses of the vmPFC to conditioned stimuli that predict primary reinforcers are reduced by devaluation of the specific primary reinforcer that they predict. This suggests that the vmPFC plays a role in predicting the future rewarding consequences of different behaviors by accessing information about their specific rewarding consequences in the past. Combined lesion and physiological studies in rodents by Schoenbaum, Setlow, Saddoris, and Gallagher (2003) have shown that this ability of the vmPFC to encode "predictive reward value" requires an intact amygdala. This is consistent with findings from human lesion studies, described earlier.
Recent functional imaging studies have also shown that the insular cortex is engaged by certain kinds of decision making. An fMRI study by Paulus, Rogalsky, Simmons, Feinstein, and Stein (2003) has shown that activity in the insular cortex is greater during high-risk decisions than it is during low-risk decisions. Furthermore, this study showed that the level of activity within the insular cortex predicted the probability of selecting a safe response following a punished response. An fMRI study by Sanfey and colleagues (Sanfey, Rilling, Aronson, Nystrom, \& Cohen, 2003) found that the insular cortex is activated when subjects evaluate the fairness of offers of money from another subject, which can be considered as an emotional process. This study found that the level of activity in the insular cortex predicts the likelihood of rejecting an unfair offer. The results of these studies suggest that the insular cortex plays a role in assessing risk and guiding behavior based upon the anticipation of emotional consequences, especially negative emotional consequences. The somatic-marker hypothesis attributes this function to the mapping of visceral states within the insular cortex, which gives rise to gut feelings of desire or aversion.

The mesolimbic dopamine system, which is hypothesized by the somatic-marker hypothesis to play a role in the unconscious biasing of action, has increasingly become a focus of attention as an area that plays a role in reward processing and decision making. Schultz, Montague, and colleagues have shown that the activity of single neurons within the mesolimbic dopamine system is increased by primary reinforcers (e.g., palatable foods), but only when these are delivered in an unpredictable fashion. Furthermore, they have found that these neurons also 
respond to stimuli that predict primary reinforcers and that these responses shift in time from occurring during the receipt of primary reinforcers to occurring at the onset of the predictive cues. Using sophisticated computational-modeling techniques, they have shown that activity within the mesolimbic dopamine system signals an error between actual and predicted reward and that such a signal can bias behavior in the direction of behaviors that are likely to lead to rewards in the future (this work is reviewed in Schultz, Dayan, \& Montague, 1997). This is consistent with the role of the mesolimbic dopamine system proposed by the somatic-marker hypothesis.

\section{APPLICATIONS OF THE SOMATIC-MARKER HYPOTHESIS}

Increasingly, the principles that were originally established by observing the decision-making deficits of patients with focal lesions are being applied to understanding a diverse range of human decision-making behaviors in which emotions play a critical role. For example, Greene and his colleagues (Greene, Sommerville, Nystrom, Darley, \& Cohen, 2001) have used fMRI to examine the neural systems that enable moral decision making. They found that reasoning about a variety of moral dilemmas, compared to reasoning about nonmoral dilemmas, activates a network of structures that include the vmPFC. Furthermore, this activation is greater when the moral decision involves negative consequence for another person, compared to when it involves no negative consequence for another person. This finding suggests that moral decisions, compared to nonmoral decisions, engage emotions, especially when one is required to consider the consequences of one's actions for another's well-being.

The somatic-marker framework has also been applied to understanding the decision-making impairments that are associated with drug addiction. Substance abusers show real-life decision-making impairments that are similar to those of patients with vmPFC damage. Studies by Bechara et al. (reviewed in Bechara, 2005) have shown that the performance of substance abusers on the Iowa Gambling Task is similar to that of patients with vmPFC damage. This suggests that drug addiction may be promoted in part by a dysfunction of the vmPFC whereby information about the negative emotional consequences of drug abuse cannot be used to motivate quitting. Such studies may provide important insights into how to treat substance dependence.

\section{FUTURE DIRECTIONS}

Much of the work on the neuroscience of decision making has lent support to the neuroanatomical framework originally put forth in the somatic-marker hypothesis. However, some components of this framework still remain to be addressed. For ex- ample, it will be important to examine the extent to which the sensory feedback of emotional/bodily states within regions such as the mesolimbic dopamine system and the insular cortex influences both conscious gut feelings and nonconscious biasing of behavior. It will also be important to see how the areas implicated in this theory work together to facilitate decision making. This may be aided by computational models of decision making (e.g., Yechiam, Busemeyer, Stout, \& Bechara, 2005) that generate predictions about how these areas will be activated under different conditions of reward, uncertainty, and risk, and how lesions in these regions will affect different components of the decision-making process. A further question regards the role of neurotransmitter systems, such as the mesolimbic dopamine system, in decision making (see Robbins, 2000, for a review of work on pharmacologic manipulations of decision making). These studies may shed light on how drug therapies can be used to treat the decision-making impairments associated with certain mental illnesses.

In general terms, the somatic-marker hypothesis provides a basis for understanding how the most elevated of human abilities - the capacity to make decisions in the moral, social, and financial realms-are related to basic motivational and homeostatic processes that are shared among all mammalian species. The theory serves as a launching point for understanding not only decision making but also a variety of goaldirected processes in which affect and motivation are integrated with the planning of complex action.

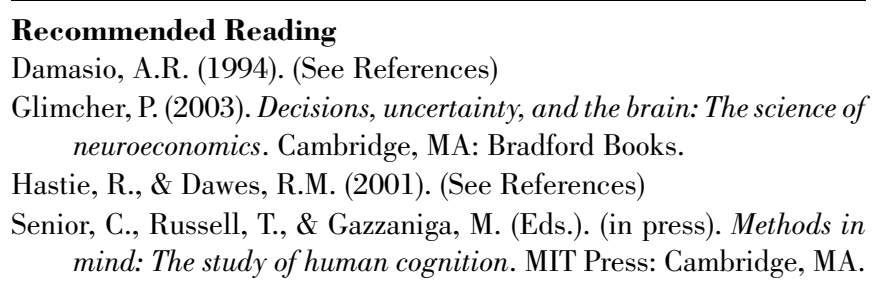

Acknowledgments - The decision-neuroscience research of Antoine Bechara is supported by National Institute on Drug Abuse Grants DA11779-02, DA12487-03, DA16708, and by National Institute of Neurological Disorders and Stroke Grant NS19632-23, and that of Baba Shiv is supported by National Science Foundation Grant SES 03-50984.

\section{REFERENCES}

Bechara, A. (2005). Decision making, impulse control and loss of willpower to resist drugs: A neurocognitive perspective. Nature Neuroscience, 8, 1458-1463.

Bechara, A., \& Damasio, A. (2005). The somatic marker hypothesis: A neural theory of economic decision-making. Games and Economic Behavior, 52, 336-372. 
Damasio, A.R. (1994). Descartes' error: Emotion, reason and the human brain. New York: Putnam and Sons.

Gottfried, J.A., O'Doherty, J., \& Dolan, R.J. (2003). Encoding predictive reward value in human amygdala and orbitofrontal cortex. Science, 301, 1104-1107.

Greene, J.D., Sommerville, R.B., Nystrom, L.E., Darley, J.M., \& Cohen, J.D. (2001). An fMRI investigation of emotional engagement in moral judgment. Science, 293, 2105-2108.

Hastie, R., \& Dawes, R.M. (2001). Rational choice in an uncertain world. Thousand Oaks, CA: Sage Publications.

Kahneman, D., \& Tversky, A. (1979). Prospect theory: An analysis of decision under risk. Econometrica, 47, 263-291.

Paulus, M.P., Rogalsky, C., Simmons, A., Feinstein, J.S., \& Stein, M.B. (2003). Increased activation in the right insula during risk-taking decision making is related to harm avoidance and neuroticism. Neuroimage, 19, 1439-1448.

Rahman, S., Sahakian, B.J., Cardinal, R.N., Rogers, R.D., \& Robbins, T.W. (2001). Decision making and neuropsychiatry. Trends in Cognitive Science, 5, 271-277.
Robbins, T.W. (2000). Chemical neuromodulation of frontal-executive functions in humans and other animals. Experimental Brain Research, 133, 130-138.

Rolls, E.T. (2004). The functions of the orbitofrontal cortex. Brain and Cognition, 55, 11-29.

Sanfey, A.G., Rilling, J.K., Aronson, J.A., Nystrom, L.E., \& Cohen, J.D. (2003). The neural basis of economic decision-making in the ultimatum game. Science, 300, 1755-1758.

Schoenbaum, G., Setlow, B., Saddoris, M.P., \& Gallagher, M. (2003). Encoding predicted outcome and acquired value in orbitofrontal cortex during cue sampling depends upon input from basolateral amygdala. Neuron, 39, 855-867.

Schultz, W., Dayan, P., \& Montague, P.R. (1997). A neural substrate of prediction and reward. Science, 275, 1593-1599.

Yechiam, E., Busemeyer, J.R., Stout, J.C., \& Bechara, A. (2005). Using cognitive models to map relations between neuropsychological disorders and human decision-making deficits. Psychological Science, 16, 973-978. 